\title{
EQUALITY OF SPECTRA OF QUASI-SIMILAR HYPONORMAL OPERATORS 1
}

\author{
STUART CLARY
}

\begin{abstract}
It is shown that quasi-similar hyponormal operators have equal spectra.
\end{abstract}

If $H$ and $K$ are (complex) Hilbert spaces, the (bounded linear) operator $X: H \rightarrow K$ is said to be quasi-invertible iff it is one-to-one and has dense range. Two operators $A: H \rightarrow H$ and $B: K \rightarrow K$ are quasi-similar provided there exist quasi-invertible operators $X: H \rightarrow K$ and $Y: K \rightarrow H$ such that $X A=B X$ and $Y B=A Y$. Quasi-similarity was first introduced by Sz.-Nagy and Foias (see, for example, [5]) in their theory of an infinite dimensional analogue of the Jordan form for certain classes of operators; it replaces the familiar notion of similarity which is the appropriate equivalence relation to use with finite dimensional Jordan forms. Quasi-similarity is the same thing as similarity in finite dimensional spaces, but in infinite-dimensional spaces it is a much weaker relation, so weak that two operators can be quasi-similar and yet have unequal spectra [5, p. 262], [4, p. 683].

For normal operators this cannot happen: it follows from the PutnamFuglede commutativity theorem that if two normal operators are quasisimilar, they are actually unitarily equivalent [2, Lemma 4.1, p. 23], and therefore have equal spectra. This result does not generalize to pairs of hyponormal operators, for D. E. Sarason has given an example of two hyponormal operators which are similar but not unitarily equivalent [3, Solution 156, p. 309]. The following elaboration on Sarason's example shows that quasi-similar hyponormal operators need not even be similar. (Recall that $B: K \rightarrow K$ is hyponormal iff $B B^{*} \leq B^{*} B$ in the partial order for Hermitian operators, or equivalently iff $\left\|B^{*} g\right\| \leq\|B g\|$ for all $g$ in $K$. )

Let $U$ denote the unweighted unilateral shift of multiplicity 1 , and let $S_{n}$ be the unilateral weighted shift with weights $1 / n, 1,1,1, \ldots$ Let $U_{\infty}=U \oplus U \oplus U \oplus \cdots$ and $S=S_{1} \oplus S_{2} \oplus S_{3} \oplus \ldots$. Each $S_{n}$ is similar to $U$, so $U_{\infty}$ and $S$ are quasi-similar by [4, Theorem 2.5, p. 681], and they are easily seen to be hyponormal (both are actually subnormal [3, Chapter

\footnotetext{
AMS (MOS) subject classifications (1970). Primary 47B20; Secondary 47A10.

Key words and phrases. Hyponormal operator, paranormal operator, quasisimilarity, spectrum.

1 This note is part of a Universtiy of Michigan doctoral dissertation written under the direction of R. G. Douglas.
}

Received by the editors October 17, 1973 and, in revised form, September 27, 1974. 
16]). But $U_{\infty}$ is an isometry and $S$ is not bounded below, so they are not similar. Furthermore, they have unequal approximate point spectra.

The purpose of this note is to show that, in spite of such examples, quasi-similar hyponormal operators always have equal spectra. The proof is based on elementary in equalities contained in the following two lemmas.

Lemma A. Let $B: K \rightarrow K$ be hyponormal and let $\left\{g_{n}\right\}_{n=0}^{\infty}$ be a sequence in $K$ such that $B g_{n+1}=g_{n}$ for all $n \geq 0$. Then either $\left\|g_{0}\right\| \geq\left\|g_{1}\right\| \geq\left\|g_{2}\right\| \geq$ . or $\left\|g_{n}\right\| \rightarrow \infty$ as $n \rightarrow \infty$.

Proof. For any $g$ in $K$,

$$
\begin{aligned}
\|B g\| & =(B g, B g)^{1 / 2}=\left(B^{*} B g, g\right)^{1 / 2} \leq\left(\left\|B^{*} B g\right\|\|g\|\right)^{1 / 2} \\
& \leq\left(\left\|B^{2} g\right\|\|g\|\right)^{1 / 2} \leq 1 / 2\left(\left\|B^{2} g\right\|+\|g\|\right) .
\end{aligned}
$$

Letting $g=g_{n+2}$ we see that $\left\|g_{n+1}\right\| \leq 1 / 2\left(\left\|g_{n}\right\|+\left\|g_{n+2}\right\|\right)$, so the sequence $\left\{\left\|g_{n}\right\|\right\}$ is convex and the conclusion follows at once.

Lemma B. Suppose $A: H \rightarrow H$ is invertible, $B: K \rightarrow K$ is hyponormal, and $X: H \rightarrow K$ satisfies $X A=B X$. Then $\left\|X A^{-1} f\right\| \leq\left\|A^{-1}\right\|\|X f\|$ for all $f$ in $H$.

Proof. Assume with no loss of generality that $\operatorname{dim} H \geq 1$, and let $c=$ $\left\|A^{-1}\right\|>0$. Fix $f$ in $H$ and define $g_{n}=c^{-n} X A^{-n} f$ for $n \geq 0$. Then $c B g_{n+1}=g_{n}$ and $\left\|g_{n}\right\| \leq\|X\|\|f\|$ for all $n \geq 0$. Since $c B$ is hyponormal, $\left\|g_{0}\right\| \geq\left\|g_{1}\right\| \geq\left\|g_{2}\right\| \geq \cdots$ by Lemma $A$, and the first inequality in this chain, $\left\|g_{1}\right\| \leq\left\|g_{0}\right\|$, shows that $\left\|X A^{-1} f\right\| \leq\left\|A^{-1}\right\|\|X f\|$.

Theorem 1. If $A: H \rightarrow H$ is invertible, $B: K \rightarrow K$ is hyponormal, and $X:$ $H \rightarrow K$ has dense range and satisfies $X A=B X$, then $B$ is invertible.

Proof. Again assume $\operatorname{dim} H \geq 1$. Clearly the range of $B$ contains the range of $X$, so $B$ has dense range. It remains only to show that $B$ is bounded below, and by continuity it will suffice to show that $B$ is bounded below on the range of $X$. But that is easy: the in equality $\left\|B\left(X f_{1}\right)\right\| \geq\left\|X f_{1}\right\| /\left\|A^{-1}\right\|$ follows from Lemma $\mathrm{B}$ if we set $f_{1}=A^{-1} f$.

Theorem 2. Quasi-similar hyponormal operators have equal spectra.

Proof. If $A$ and $B$ are quasi-similar hyponormal operators, then, for any complex number $\lambda, A-\lambda$ and $B-\lambda$ are also quasi-similar and hyponormal, so by Theorem 1 they are both invertible or both noninvertible. Thus the spectrum of $A$ is the same as that of $B$. (Note that the injectivity of the interwining maps was never used.)

Theorem 1 and both lemmas remain true if we assume only that $B$ is paranormal (defining condition: $\|B g\|^{2} \leq\left\|B^{2} g\right\|\|g\|$ for all $g$ in $K$ ) rather 
than hyponormal. (Every hyponormal operator is paranormal, but not conversely.) I do not know whether quasi-similar paranormal operators necessarily have equal spectra. The proof of Theorem 2 would generalize to prove this if the sum of a paranormal operator and a scalar multiple of the identity were always paranormal; however, it is known that this is not so [1, p. 174].

Another question I cannot answer concerns the Fredholm essential spectrum: Must quasi-similar hyponormal operators have equal essential spectra?

\section{REF ERENCES}

1. T. Ando, Operators with a norm condition, Acta Sci. Math. (Szeged) 33 (1972), 169-178. MR $47 \# 9334$.

2. R. G. Douglas, On the operator equation $S^{*} X T=X$ and related topics, Acta Sci. Math. (Szeged) 30 (1969), 19-32. MR 40 \#3347.

3. P. R. Halmos, A Hilbert space problem book, Van Nostrand, Princeton, N.J., 1967. MR $34 \# 8178$.

4. T. B. Hoover, Quasi-similarity of operators, Illinois J. Math. 16 (1972), 678686. MR $47 \# 866$.

5. B. Sz.-Nagy and C. Foiaş, Analyse harmonique des opérateurs de l'espace de Hilbert, Masson, Paris; Akad. Kiadó, Budapest, 1967; English rev. transl., NorthHolland, Amsterdam; American Elsevier, New York; Akad. Kiadó, Budapest, 1970. MR $37 \# 778 ; 43 \# 947$.

DEPARTMENT OF MATHEMATICS, CITY COLLEGE (CUNY), NEW YORK, NEW YORK 10031 